\title{
Different Geometries of Superheterodyne Amplification of Electromagnetic Beams in Waveguides Nitride-Dielectric
}

\author{
Jesus Escobedo-Alatorre, Volodymyr Grimalsky, Svetlana Koshevaya, \\ Margarita Tecpoyotl-Torres
}

Institute for Basic and Applied Research (IICBA), Center for Investigations on Engineering and Applied Science (CIICAp), Autonomous University of State Morelos (UAEM), Cuernavaca, Mexico

Email: jescobedo@uaem.mx,v_grim@yahoo.com,svetlana@uaem.mx, tecpoyotl@uaem.mx

How to cite this paper: Escobedo-Alatorre, J., Grimalsky, V., Koshevaya, S. and Tecpoyotl-Torres, M. (2020) Different Geometries of Superheterodyne Amplification of Electromagnetic Beams in Waveguides Nitride-Dielectric. Journal of Electromagnetic Analysis and Applications, 12, 159-168. https://doi.org/10.4236/jemaa.2020.121101 $\underline{3}$

Received: August 26, 2020

Accepted: November 27, 2020

Published: November 30, 2020

Copyright $\odot 2020$ by author(s) and Scientific Research Publishing Inc. This work is licensed under the Creative Commons Attribution International License (CC BY 4.0).

http://creativecommons.org/licenses/by/4.0/

\begin{abstract}
The superheterodyne amplification of electromagnetic waves is investigated when the resonant three-wave interaction of two electromagnetic waves with the space charge wave occurs in the waveguides nitride $n$-GaN, $n$-InN films-dielectric. The amplification of SCW waves due to the negative differential conductivity is investigated in nitride $n-\mathrm{GaN}, n-\mathrm{InN}$ films at the frequencies $f \leq 400 \mathrm{GHz}$ in the lower part of the terahertz $(\mathrm{THz})$ range. The electromagnetic waves are either in the upper part of $\mathrm{THz}$ range or in the optical range. The superheterodyne amplification is considered in two geometries, the collinear one in which the three interacting waves travel in the same direction and the anti-collinear geometry where the second electromagnetic wave propagates in the opposite direction. The preferences and drawbacks of each geometry are pointed out. The finite width of space charge waves leads to decrease of increments of amplification.
\end{abstract}

\section{Keywords}

Superheterodyne Amplification, Negative Differential Conductivity, Space Charge Wave, Nitride Films, Terahertz Range

\section{Introduction}

The electromagnetic radiation of terahertz range $(\mathrm{THz})$ is widely used in spectroscopy, medicine, scanning, and environmental science [1]. Now the sources of the $\mathrm{THz}$ radiation are both electron tubes (gyrotrons, backward wave tubes) and lasers. These sources are not well compatible with the traditional millimeter 
wave devices. An alternative way can be realized with the amplification of electromagnetic waves (EMW) under three-wave resonant interactions in semiconductor films where the negative differential conductivity (NDC) occurs. This method is based on the transfer of amplification of a space charge wave (SCW) at the microwave range or the lower part of $\mathrm{THz}$ range [2] to EMW at higher frequencies [3] [4]. EMW are either in the upper part of $\mathrm{THz}$ range or in the optical range, including infrared and visible ones.

Generally for the practical needs in the active media the transfer of the linear amplification of a low-frequency wave to high-frequency one is important [4]-[9], i.e. the superheterodyne amplification. A preference of the superheterodyne amplification is the using of relatively low levels of the electromagnetic pump wave at the intermediate frequency, which is utilized for the resonant wave coupling.

In this paper, different geometries of the superheterodyne amplification of EMW are investigated. The superheterodyne amplification of EM waves can realize in waveguides based on nitride $n$-GaN, $n$-InN films where the three-wave resonant interaction of two EMW with SCW amplified due to NDC can be realized. The superheterodyne amplification takes place both in the collinear geometry, where the propagating EM modes and the SCW propagate in the same direction, and in the anti-collinear one, where the pump EMW at the intermediate frequency propagates in the opposite direction. This amplification is due to the transfer of amplification from SCW to the signal EMW at the highest frequency and is effective, namely, the values of $20-40 \mathrm{~dB}$ can be reached at the lengths of $40-100 \mu \mathrm{m}$. An influence of the finite widths of EM waves in the plane of the film has been taken into account.

\section{Model and Equations}

It is considered $n$-GaN or $n$-InN film of a submicron thickness placed onto a dielectric substrate, see Figure 1. The nitride film is at $0<x<2 l$, a dielectric substrate is at $x<0$. Above at $x>2 I$ there is either vacuum or a dielectric. Below the symmetric waveguide is considered, so above there is the same dielectric as at $X$ $<0$.

The nitride film is the waveguide for EM waves when the condition is satisfied: $\varepsilon_{2 E M}>\varepsilon_{1 E M}, \varepsilon_{3 E M}$. Here $\varepsilon_{1,2,3 E M}$ are corresponding permittivities in the optical range or upper part of $\mathrm{THz}$ one, which differ from their values in the microwave range due to the frequency dispersion [10] [11] [12] [13]. In such a dielectric waveguide it is possible to realize the resonant three-wave interaction of the following waves localized along $O X$ axis: forward EMW at the frequency $\omega_{1}$ and the longitudinal wave number $k_{1}$, backward EMW $\omega_{2}, k_{2}$, and SCW $\omega_{3}, k_{3}$. The resonant matching conditions are (see Figure 1):

$$
\begin{aligned}
& \omega_{3}=\omega_{1}-\omega_{2}, \\
& k_{3}=k_{1}-k_{2} \text { for the collinear geometry; } \\
& k_{3}=k_{1}+\left|k_{2}\right| \text { for anti-collinear one. }
\end{aligned}
$$


(a)

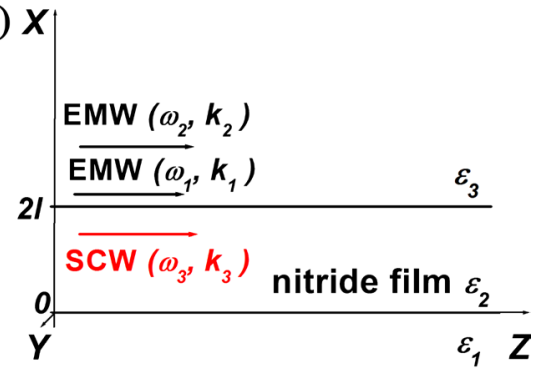

(b) $x$

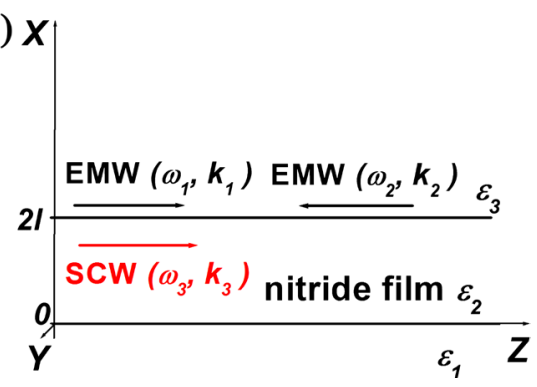

Figure 1. Geometry of the problem. The region $0<x<2 l$ is a nitride film, $x<0$ is a dielectric substrate, $x>2 l$ is either vacuum or dielectric. The interacting waves are two EM waves $\left(\omega_{1}, k_{1}\right),\left(\omega_{2}, k_{2}\right)$ and a space charge wave $\left(\omega_{3}, k_{3}\right)$. The waves are non-uniform along $O Y$ axis. The bias constant electric field is aligned along $O Z$ axis, SCW propagates also along $O Z$. Part a) is the collinear geometry and b) is anti-collinear one.

The frequencies of EMW and SCW are of about: $\omega_{1,2} \sim 10^{14}-4 \times 10^{15} \mathrm{~s}^{-1}$, $\omega_{3} \approx 2 \omega_{1} \times\left(v_{0} \varepsilon_{2 E M}^{1 / 2} / c\right) \sim 10^{12}-4 \times 10^{12} \mathrm{~s}^{-1} \quad\left(f_{3}=\omega_{3} / 2 \pi \sim 150-700 \mathrm{GHz}\right)$ in the anti-collinear geometry. In the collinear geometry the SCW frequency is 3 - 5 times lower, it is $\omega_{3}=v_{0} \cdot\left(k_{1}-k_{2}\right) \equiv\left(\varepsilon_{\omega_{1}}^{1 / 2}-\varepsilon_{\omega_{2}}^{1 / 2}\right) \cdot \omega_{1} \cdot v_{0} / c$. Here

$v_{0} \approx 2.4 \times 10^{7} \mathrm{~cm} / \mathrm{s}$ is the velocity of SCW in $n-G a N$ [10] [11], $\varepsilon_{\omega_{1,2}}$ are effective permittivities of EM waveguide modes at the frequencies $\omega_{1,2}$. For the collinear geometry is rather better to choose the lowest (fundamental) EM mode at the frequency $\omega_{1}$ and the third EM mode at $\omega_{2}$.

The dynamics of SCW is described by the equations of motion of the electron fluid jointly with the Poisson equation for the electric field. At the frequency range $f \leq 400 \mathrm{GHz}$ the simplest diffusion-drift equation can be applied:

$$
\begin{aligned}
& \frac{\partial n}{\partial t}+\operatorname{div}(\boldsymbol{v}(E) n-D \nabla n)=0, \quad \boldsymbol{v}=\mu(|E|) \boldsymbol{E} ; \\
& \operatorname{div}\left(\varepsilon_{0} \varepsilon(x) \nabla \tilde{\varphi}\right)=-e\left(n-n_{0}\right), \quad \boldsymbol{E}=-\nabla \tilde{\varphi}+E_{0}
\end{aligned}
$$

Here $n$ is the electron concentration, $\tilde{\varphi}$ is the potential of the variable electric field, $v$ is the electron drift velocity, $n_{0}$ is the equilibrium electron concentration, which is equal to the donor one; $D$ is the diffusion coefficient, $\mu(E)$ is the electron mobility that depends on the value of the electric field, $E_{0}$ is a bias constant electric field.

The data for nitrides GaN, InN are taken from [10] [11]. The coordinate frame is aligned along the crystalline axes. The lower indices 1, 2, 3 are related to the substrate, film, and the region over the film. The corresponding dielectric permittivities in the microwave range are $\varepsilon_{1}, \varepsilon_{2}, \varepsilon_{3}$. 
For EMW the Maxwell equations are:

$$
\begin{aligned}
& \nabla \times \boldsymbol{H}=\varepsilon_{0} \varepsilon(x) \frac{\partial \boldsymbol{E}}{\partial t}+\boldsymbol{j}, \quad \boldsymbol{j}=e\left(n_{0}+\tilde{n}\right) \boldsymbol{v} \\
& \nabla \times \boldsymbol{E}=-\left(1 / \varepsilon_{0} c^{2}\right) \frac{\partial \boldsymbol{H}}{\partial t}, \\
& \boldsymbol{B}=\mu_{0} \boldsymbol{H}, m^{*} \frac{\partial \boldsymbol{v}}{\partial t} \approx e \boldsymbol{E} .
\end{aligned}
$$

Here $m^{*}$ is the effective electron mass, $\tilde{n}$ is the variable electron concentration of SCW; $\boldsymbol{v}$ is the high frequency electron velocity.

The influence on EMW on SCW is due to taking into account the Lorentz force in Equation (2), i.e. due to the substitution there

$$
E_{z} \rightarrow E_{z}^{\prime}=E_{z}-\mu_{0} v_{y} H_{x} .
$$

\section{Linear Amplification of Space Charge Waves in Nitride Films}

Here the linear amplification of SCW due to NDC is considered. At the surfaces of the film the following boundary conditions are used for the density of the electric current $j[2]$ :

$$
j_{x}(x=0)=0, j_{x}(x=2 l)=0 ; \boldsymbol{j}=e(\boldsymbol{v}(E) n-D \nabla n) .
$$

Equation (2) have been linearized, and the solutions for the perturbation of the electron concentration $\tilde{n}$ and for the variable part of the electric potential $\tilde{\varphi}$ are searched as the travelling wave $\tilde{n}, \tilde{\varphi} \sim \exp \left(i \omega t-i k_{z} z-i k_{y} y\right)$. Now an attention is paid to the transversely non-uniform case $k_{y} \neq 0$. The dispersion equation for SCW $k_{z}=k\left(\omega, k_{y}\right)$ has been got from the substitution of the solutions within each partial region into the boundary conditions (5) and standard electric boundary conditions. In the case of spatial amplification of SCW, when a frequency $\omega$ and a transverse wave number $k_{y}$ are real $(\omega>0)$, the longitudinal wave number is complex, $k_{z}=k_{z}^{\prime}+i k_{z}^{\prime \prime}\left(k_{z}^{\prime}>0\right)$; within a certain frequency range there exists the imaginary part $k_{z}^{\prime \prime}>0$. The case of NDC is under consideration: $\mathrm{d} v / \mathrm{d} E<0$.

The spatial increment $k_{z}^{\prime \prime}$ depends essentially on the value of $k_{y}$. From the linearized equations it is possible to write down:

$$
k_{z}^{\prime \prime}\left(\omega, k_{y}\right) \approx k^{\prime \prime}(\omega, 0)-g k_{y}^{2} ; g \approx 2 l \frac{\omega_{M}}{\omega} \frac{\varepsilon_{2}}{\varepsilon_{1}+\varepsilon_{3}}
$$

Here $\omega_{M}=e n_{0} \mu / \varepsilon_{0} \varepsilon_{2}$ is the Maxwellian relaxation frequency, $\mu=\mu\left(E_{0}\right)=v_{0} / E_{0} ; v_{0}=\mu\left(E_{0}\right) E_{0}$ is the constant part of the drift velocity. Therefore, a relatively small transverse non-uniformity of the beam of SCW $\leq 5$ $\mu \mathrm{m}$ results in the essential decrease of the amplification, because the value of the relaxation frequency $\omega_{M} \sim 10^{13} \mathrm{~s}^{-1}$ is high.

The results for $n$-GaN film with different thicknesses 21 and electron concentrations $n_{0}$ for the bias electric field $E_{0}=150 \mathrm{kV} / \mathrm{cm}$ are presented in Figure 2 for the case of transversely uniform SCW $k_{y}=0$. The curve 1 is for $2 l=0.5 \mu \mathrm{m}$, 


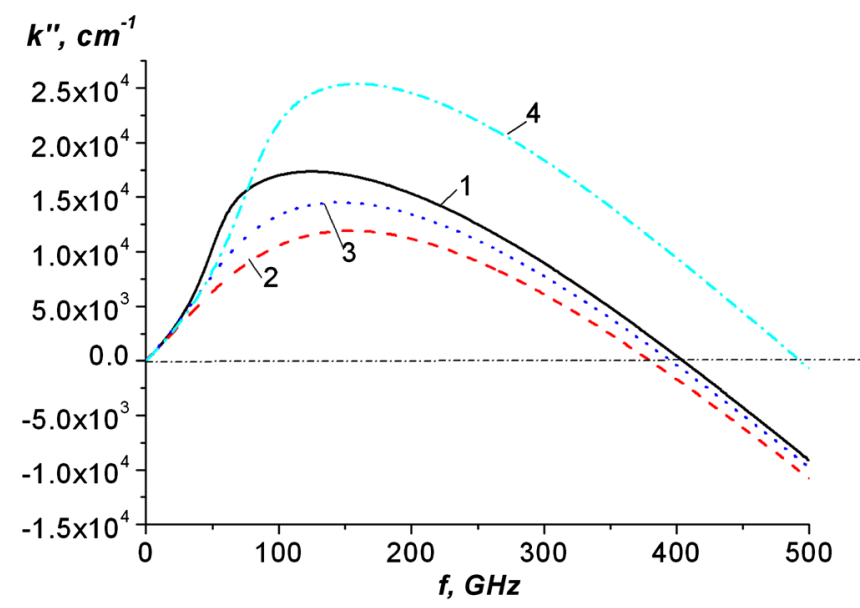

Figure 2. Dependence of spatial increments of amplification of SCW on frequency in different $n-\mathrm{GaN}$ films.

$n_{0}=2 \times 10^{17} \mathrm{~cm}^{-3}, \quad \varepsilon_{1}=\varepsilon_{3}=3.9\left(\mathrm{SiO}_{2}\right.$ above and below the film); the curve 2 is for $2 l=0.5 \mu \mathrm{m}, n_{0}=2 \times 10^{17} \mathrm{~cm}^{-3}, \varepsilon_{1}=\varepsilon_{3}=8.5(\mathrm{AlN})$; the curve 3 is for $2 l=0.7 \mu \mathrm{m}, \quad n_{0}=2 \times 10^{17} \mathrm{~cm}^{-3}, \varepsilon_{1}=\varepsilon_{3}=8.5(\mathrm{AlN}) ;$ the curve 4 is for $2 l=0.5 \mu \mathrm{m}, n_{0}=3 \times 10^{17} \mathrm{~cm}^{-3}, \varepsilon_{1}=\varepsilon_{3}=8.5(\mathrm{AlN})$. Analogous results have been obtained for the waveguides on the base of $n$-InN films, where the increments of amplification are even greater.

Some parameters that are used below to investigate the superheterodyne amplification are: the frequency range is $f \leq 400 \mathrm{GHz}$, the spatial increment is of about $k_{z}^{\prime \prime} \approx 2 \times 10^{4} \mathrm{~cm}^{-1}$ for the frequency $f \approx 200 \mathrm{GHz}$.

\section{The Equations for Three-Wave Interaction}

The nonlinear interaction is due to the modulation of the permittivity in the optical range (at higher frequencies) by SCW and due to the ponderomotive action of EMW to SCW in the microwave range (at lower frequencies). In the case of moderate nonlinearity it is possible to describe this resonant interaction by means of slowly varying wave amplitudes [3] [4] [5] [8] [12] [14].

It is investigated the interaction of transverse electric (TE) EM modes: $E=E_{y}$ the magnetic field is $H=\left(H_{x}, 0, H_{z}\right)$. For the transverse magnetic modes the results are similar.

At the boundaries of the film the standard EM boundary conditions are applied. For SCW the condition of the absence of the surface space charge leads to the conditions (5).

The solutions of Equations (2) and (3) for SCW and for EM waves are searched in the form:

$$
\begin{aligned}
& \begin{aligned}
E(x, y, z, t)= & \frac{1}{2}\left(F_{1}(x) A_{1}(z, t, y) \exp \left(i\left(\omega_{1} t-k_{1} z\right)\right)\right. \\
& \left.+F_{2}(x) A_{2}(z, t, y) \exp \left(i\left(\omega_{2} t-k_{2} z\right)\right)\right)+ \text { c.c.; }
\end{aligned} \\
& \tilde{n}=-\frac{i n_{0}}{2} U(z, t, y) F_{3}(x) \exp \left(i\left(\omega_{3} t-k_{3} z\right)\right)+\text { c.c. }
\end{aligned}
$$


Here $A_{1,2}(z, t, y), U(z, t, y)$ are slowly varying amplitudes for EMW and SCW, $F_{1,2}(x), F_{3}(x)$ are linear transverse profiles of the waves. The pump wave is $A_{2} ; A_{1}$ is EM pulse under amplification, $U$ is SCW, which is produced under the three-wave interaction.

When using the orthogonality of waveguide modes [13], it is possible to get the coupled equations for the slowly varying amplitudes:

$$
\begin{gathered}
\frac{\partial U}{\partial t}+\frac{\partial U}{\partial z}-\Gamma_{U} U \cdot\left(1-|U|^{2}\right)-g_{U} \frac{\partial^{2} U}{\partial y^{2}}=i A_{1} A_{2}^{*} ; \\
\frac{\partial A_{1}}{\partial z}+i g_{1} \frac{\partial^{2} A_{1}}{\partial y^{2}}+\Gamma_{1} A_{1}=-A_{2} U ; \\
\text { collinear geometry }: \frac{\partial A_{2}}{\partial z}+i g_{2} \frac{\partial^{2} A_{2}}{\partial y^{2}}+\Gamma_{2} A_{2}=A_{1} U^{*} ; \\
\text { anti-collinear geometry }: \frac{\partial A_{2}}{\partial z}-i g_{2} \frac{\partial^{2} A_{2}}{\partial y^{2}}-\Gamma_{2} A_{2}=-A_{1} U^{*} .
\end{gathered}
$$

Equation (8) are presented in the non-dimensional form. The unity for EMW amplitudes corresponds to the intensity of about $10 \mathrm{MW} / \mathrm{cm}^{2}$. The temporal scale $t_{n}$ is related to the motion of SCW, i.e. $t_{n}=I_{n} / V_{0}$, where $I_{n}$ is the spatial scale, $V_{0}$ is the velocity of SCW. Here the dissipation coefficients for EM modes have been introduced $\Gamma_{1,2} ; \Gamma_{U}>0$ is the increment of spatial amplification of SCW that has been considered for the case $k_{y}=0$. Also the saturation of amplification of SCW is taken into account when the maximum values of SCW are close to $n_{0}$, i.e. at $|U| \leq 1$.

Because the nonlinear interaction of the waveguide modes is considered, the efficiency of the interaction is determined by the overlap integral $S$ [13] of the interacting EM modes and SCW. The overlap integral is $S \approx 1$ for the anti-collinear interaction and $S \approx 0.4$ for collinear one, because under the anti-collinear interaction it is possible to choose the same fundamental modes for EMW at the frequencies $\omega_{1,2}$, whereas the interacting modes should be different for the collinear interaction.

One can see that the transverse nonuniformity along $O Y$ axis of EMW leads to the wave diffraction [9] [14], whereas the nonuniformity of SCW appears like the diffusion. The mechanism of the wave coupling is as follows. SCW modulates the effective permittivity of EM waves; the influence of EM waves on SCW is ponderomotive, due to the term Equation (4).

The boundary conditions for the slowly varying amplitudes are:

$$
\begin{aligned}
& U(z=0, t, y)=0 ; \\
& A_{1}(z=0, t, y)=A_{10} \Phi(t) \Psi(y) ; \\
& \text { the collinear geometry: } A_{2}(z=0, t, y)=A_{20} \Psi(y) \\
& \text { the anti-collinear geometry: } A_{2}\left(z=L_{z}, t, y\right)=A_{20} \Psi(y) .
\end{aligned}
$$

Here $A_{10}$ is a maximum amplitude of the small input pulse at the frequency $\omega_{1}$, $\Phi(t)$ is the temporal shape of the pulse, $\Psi(y)$ is its transverse shape; $A_{20}$ is the 
constant amplitude of the EM pump wave; $A_{10} \ll A_{20}$. The length of the system is $L_{z}$. The width of the film is $L_{y}$ it is quite big to avoid an influence of the boundaries along $O Y$ axis. The temporal shape of the input signal pulse $A_{1}$ is chosen as Gaussian-like, the transverse shape of EMW $\Psi(y)$ is bell-like with the half-width of about $y_{0} \geq 5 \mu \mathrm{m}$.

\section{Superheterodyne Amplification of Electromagnetic Pulses}

It is investigated the superheterodyne amplification of small input pulses of EMW at the carrier frequency $\omega_{1}$, i.e. the transfer of amplification of SCW due to NDC to EMW at higher frequency $\omega_{1}$ in the presence of the pump EMW at the frequency $\omega_{2}$.

The mechanism of the superheterodyne amplification is as follows [3] [4]. Because of the three-wave interaction, the mixing of two EMW results in the generation of SCW at lower frequency $\omega_{3}$. Then SCW is amplified in a medium with NDC. In that turn, in the output of the system the amplified EM wave appears at the frequency $\omega_{1}$. The lengths of the waveguides are $L_{z}=40-100 \mu \mathrm{m}$.

The difference schemes are used to approximate Equationi (8). The splitting with respect to physical factors has been used to take into account the wave diffusion term with $\partial^{2} U / \partial y^{2}$ in the equation for $U$ [15] [16]. The upwind difference approximations have been applied for EM amplitudes $A_{1,2}$; for the diffraction terms the implicit difference approximation has been used.

The typical results of simulations are presented in Figure 3. The part a) is for the anti-collinear geometry, the part b) is for collinear one. The waveguide $\mathrm{SiO}_{2}-$ $n$-GaN $-\mathrm{SiO}_{2}$ is considered, see Figure 2, curve 1. In the anti-collinear geometry the signal EM frequency is $\omega_{1} \approx 3 \times 10^{14} \mathrm{~s}^{-1}$, SCW frequency is $\omega_{3} \approx 1.5 \times 10^{12} \mathrm{~s}^{-1}$. In the collinear geometry they are $\omega_{1} \approx 2 \times 10^{15} \mathrm{~s}^{-1}$, SCW frequency is $\omega_{3} \approx 1.5 \times 10^{12} \mathrm{~s}^{-1}$. The input amplitude for the pump is $A_{20}=0.04$, it corresponds to the input intensity of about $20 \mathrm{~kW} / \mathrm{cm}^{2}$. The length of the system is $L_{z}=100$ $\mu \mathrm{m}$. The temporal scale is $t_{n}=4 \mathrm{~ns}$ for the waveguide on the base of $n-\mathrm{GaN}$ and $t_{n}=3 \mathrm{~ns}$ for $n-\mathrm{InN}$. The duration of the input EM pulse is $t_{0}=40$ ps for $n-\mathrm{GaN}$ and $t_{0}=40$ ps for $n$-InN. The input width of EMW is $y_{0}=5 \mu \mathrm{m}$. At smaller widths an influence of wave diffraction and diffusion becomes important and reduces the efficiency of the amplification. It is seen that the superheterodyne amplification is characterized by the wide dynamic range, i.e. it is possible to amplify the input EM pulses with the intensities that differ 7 orders and more.

Both geometries, anti-collinear and collinear, are suitable for the superheterodyne amplification. But the anti-collinear geometry is preferable for the efficiency, because the overlap integral of the interacting modes is bigger. But the collinear geometry seems preferable for decreasing the corresponding resonant frequency of SCW, i.e. for the easier realization of amplification due to NDC. In another words, in the collinear geometry it is possible to amplify EM signals at higher frequencies.

It is possible to amplify EM pulses of durations $t_{0} \geq 10 \mathrm{ps}$. At smaller durations 

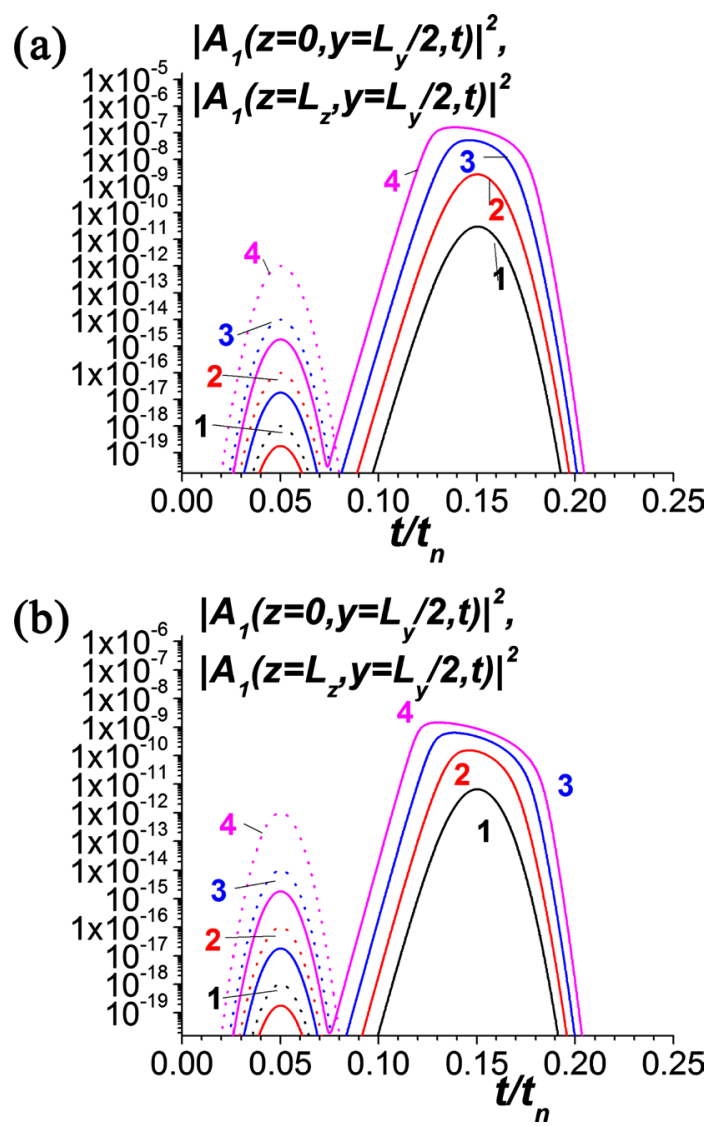

Figure 3. The superheterodyne amplification of EM pulses under different input amplitudes $A_{10}$. The input pump amplitude is $A_{20}=0.04$. Curve 1 is for $A_{10}=10^{-9}, 2$ is for $A_{10}=$ $10^{-8}, 3$ is for $A_{10}=10^{-7}$, curve 4 is for $A_{10}=10^{-6}$. The wave diffraction and diffusion are taken into account. The dot lines are the input pulses, the solid lines are the output ones. Part (a) is the anti-collinear geometry and (b) is collinear one.

the broadening of the pulses under the nonlinear interaction occurs.

When the amplitudes of input EM pulses are quite big, as ones at the curves 4 in Figure 3, the amplitudes of SCW under amplification become comparable with the equilibrium concentration, and the saturation of amplification takes place. This results in some distortion of the output EM pulse, especially near its maximum.

\section{Conclusions}

The superheterodyne amplification in the optical range and in the upper part of the terahertz range can be realized in waveguides on the base of $n$-GaN, $n$-InN films in two different geometries of the resonant three-wave interaction between two electromagnetic waves with the space charge wave. The superheterodyne amplification is due to the transfer of the amplification of the space charge wave in the medium with the negative differential conductivity to the signal electromagnetic wave. It can be realized in the waveguides of $40-100 \mu \mathrm{m}$ lengths. The input durations of electromagnetic pulses can be $10 \mathrm{ps}$ and more.

In the first geometry the propagation of the signal and pump electromagnetic 
waves is opposite, whereas in the second geometry all interacting waves propagate in the same direction. In the first geometry, the efficiency of the three-wave resonant interaction is higher than the second geometry, but possible frequencies of input optical signals can be higher in the second geometry.

The finite transverse widths of the signal and pump electromagnetic waves in the plane of the film have been taken into account. They result in the diffraction of electromagnetic modes and in the analog of the diffusion of the space charge wave. To avoid the influence of the wave diffraction and diffusion, the transverse sizes of the electromagnetic modes should be no smaller than $5 \mu \mathrm{m}$.

The dynamic range of the superheterodyne amplification without the saturation is of about 7 orders and more. The nonlinear saturation results in some distortion of the amplified pulses.

\section{Acknowledgements}

The authors thank SEP-CONACyT, Mexico, for partial support of our work.

\section{Conflicts of Interest}

The authors declare no conflicts of interest regarding the publication of this paper.

\section{References}

[1] Siegel, P.H. (2002) Terahertz Technology. IEEE Transactions on Microwave Theory and Techniques, 50, 910-928. https://doi.org/10.1109/22.989974

[2] Barybin, A.A. (1986) Waves in Thin Film Semiconductor Structures with Hot Electrons. Nauka, Moscow.

[3] Koshevaya, S., Grimalsky, V., Escobedo-Alatorre, J. and Tecpoyotl-Torres, M. (2003) Superheterodyne Amplification of Sub-Millimeter Electromagnetic Waves in an $n$-GaAs Film. Microelectronics Journal, 34, 231-235. https://doi.org/10.1016/S0026-2692(03)00003-X

[4] Kalapusha, A.L. and Kotsarenko, N.Y. (1983) Acoustoelectronic Parametric Amplification of EM Waves of Infrared and Optical Ranges in Planar Optical Waveguides. Radioelectronics and Communications Systems (Izvestiya VUZ, Radioelektronika), 26, 71-72.

[5] Weiland, J. and Wilhelmsson, H. (1977) Coherent Non-Linear Interaction of Waves in Plasmas. Pergamon Press, London.

[6] Stenflo, L. (1996) Theory of Nonlinear Plasma Surface Waves. Physica Scripta, T63, 59-62. https://doi.org/10.1088/0031-8949/1996/T63/008

[7] Rabinovich, M.I. and Fainshtein, S.M. (1973) High-Frequency Instability of an Electromagnetic Wave in a Nonequilibrium Magnetized Plasma, Journal of Experimental and Theoretical Physics, 36, 884-886.

http://jetp.ac.ru/cgi-bin/dn/e_036_05_0884.pdf

[8] Rabinovich, M.I. and Trubetskov, D.I. (1989) Oscillations and Waves: In Linear and Nonlinear Systems (Mathematics and Its Applications). Springer, Berlin.

[9] Sukhorukov, A.P. (1988) Nonlinear Wave Interactions in Optics and Radiophysics (Russian Edition). Nauka, Moscow. 
[10] Levinshtein, M.E., Rumyantsev, S.L and Shur, M.S. (2001) Properties of Advanced Semiconductor Materials: GaN, AlN, InN. Wiley-Interscience, New York. www.ioffe.ru/SVA/NSM/Semicond/GaN/

[11] Jain, S.C., Willander, M., Narayan, J. and Van Overstraeten, R. (2000) III-Nitrides: Growth, Characterization, and Properties. Journal of Applied Physics, 87, 965-1006. https://doi.org/10.1063/1.371971

[12] Chaika, G.E., Malnev, V.N. and Panfilov, M.I. (1996) Interaction of Light with Space Charge Waves. Proceedings of SPIE, 2795, 279-285. https://doi.org/10.1117/12.239220

[13] Marcuse, D. (1991) Theory of Dielectric Optical Waveguides. Academic Press, New York.

[14] Kalinovich, A., Lobanov, V.E., Sukhorukov, A.P., and Zverev, D.M. (2013) Interaction of Pulsed Laser Beams in Quadratic Nonlinear Media. Physics of Wave Phenomena, 21, 5-9. https://doi.org/10.3103/S1541308X13010020

[15] Marchuk, G.I. (1990) Splitting and Alternating Direction Methods. In: Ciarlet, P.G. and Lions, J.L., Eds., Handbook of Numerical Analysis, Elsevier, Amsterdam, 203-462. https://doi.org/10.1016/S1570-8659(05)80035-3

[16] Samarskii, A.A. (2001) The Theory of Difference Schemes. CRC Press, Boca Raton. https://doi.org/10.1201/9780203908518 